\title{
On Fully Discrete Collocation Methods for Solving Weakly Singular Integral Equations*
}

\author{
R. Kangro ${ }^{1}$ and I. Kangro ${ }^{2}$ \\ ${ }^{1}$ Institute of Mathematical Statistics, University of Tartu \\ ${ }^{2}$ Institute of Cybernetics, Tallinn University of Technology \\ ${ }^{1}$ Liivi 2-513, 50409, Tartu, Estonia \\ E-mail(corresp.): raul.kangro@ut .ee \\ ${ }^{2}$ Akadeemia 21, 12618, Tallinn, Estonia \\ E-mail: inga@cs.ioc.ee
}

Received September 30, 2008; revised October 16, 2008; published online February 25, 2009

\begin{abstract}
A popular class of methods for solving weakly singular integral equations is the class of piecewise polynomial collocation methods. In order to implement those methods one has to compute exactly certain integrals that determine the linear system to be solved. Unfortunately those integrals usually cannot be computed exactly and even when analytic formulas exist, their straightforward application may cause unacceptable roundoff errors resulting in apparent instability of those methods in the case of highly nonuniform grids. In this paper fully discrete analogs of the collocation methods, where integrals are replaced by quadrature formulas, are considered, corresponding error estimates are derived.
\end{abstract}

Key words: weakly singular, integral equation, collocation method, nonuniform grid, fully discrete method, Fredholm equation, Volterra equation.

\section{Introduction}

In many applications there arise integral equations of the form

$$
y(t)=\int_{0}^{b} K(t, s) y(s) d s+f(t),
$$

where the integration kernel $K$ is not smooth across the diagonal $t=s$. If $K(t, s) \equiv 0$ in the region $t>s$, then the equations are of Volterra type, otherwise they are of Fredholm type. A popular class of methods for solving such equations is the class of piecewise polynomial collocation methods using

\footnotetext{
* The work of Raul Kangro was partially supported by EstSF grant no. 7044. The work of Inga Kangro has been partially supported by Estonian Scientific Competence Council targeted financing grant No. 0140083s08
} 
nonuniform grids. Such methods are considered, for example, in the case of Fredholm integral equation in $[6,8,9]$ and by many others, in the case of Volterra equations in $[1,2]$ and many others; for additional references we refer to the list of references in [2]. In order to apply those methods it is necessary to compute exactly integrals of the form $\int_{t_{i}}^{t_{i+1}} K(t, s) \phi(s) d s$, where $K$ is the kernel of the integral operator and $\phi$ is a basis function corresponding to the collocation method. Unfortunately those integrals usually cannot be computed exactly and even when analytic formulas exist, their straightforward application may cause unacceptable roundoff errors resulting in apparent instability of those methods in the case of highly nonuniform grids (see [4]). Therefore it is of great practical and theoretical interest to consider methods (so called fully discrete methods), where the integrals are computed by quadrature formulas. As the kernel $K$ is not smooth, a straightforward application of quadrature formulas reduce, in general, the order of the convergence of the method. A fully discrete collocation method, preserving the order of convergence of the original collocation method, for solving Fredholm integral equations was proposed in [3], a more general class of such methods was described by E. Tamme in [7]. In the present paper we improve the estimates of E. Tamme of the error caused by the quadrature approximation of the system integrals and extend the results to other types of integral equations.

\section{Collocation Method for Solving Integral Equations}

For a given $N \in \mathbb{N}$ let $\Pi_{N}=\left\{t_{0}, t_{1}, \ldots, t_{N}: 0=t_{0}<t_{1}<\ldots<t_{N}=b\right\}$ be a partition (a mesh) of the interval $[0, b]$ (for ease of notation we suppress the index $N$ in $t_{n}=t_{n}^{(N)}$ indicating the dependence of the grid points on $N$ ).

We look for approximate solutions to integral equations in the form of piecewise polynomial functions

$$
S_{k}^{(-1)}\left(\Pi_{N}\right)=\left\{w:\left.w\right|_{\left(t_{n-1}, t_{n}\right)} \in \pi_{k}, \quad n=1, \ldots, N\right\} .
$$

Here $\pi_{k}$ denotes the set of polynomials of degree not exceeding $k$ and $\left.w\right|_{\left(t_{n-1}, t_{n}\right)}$ is the restriction of $w:[0, b] \rightarrow \mathbb{R}$ to the subinterval $\left(t_{n-1}, t_{n}\right)$.

We define $m \geq 1$ interpolation points in every subinterval $\left[t_{n-1}, t_{n}\right]$ $(n=1, \ldots, N)$ of the grid $\Pi_{N}$ by

$$
t_{n j}=t_{n-1}+\eta_{j} h_{n}, \quad j=1, \ldots, m \quad(n=1, \ldots, N),
$$

where $h_{n}=t_{n}-t_{n-1}$ and $\eta_{1}, \ldots, \eta_{m}$ are some fixed parameters (called collocation parameters) which do not depend on $n$ and $N$ and satisfy

$$
0 \leq \eta_{1}<\ldots<\eta_{m} \leq 1 .
$$

We look for an approximate solution $u$ to the solution $y$ of equation (1.1) in $S_{m-1}^{(-1)}\left(\Pi_{N}\right), m, N \in \mathbb{N}$. We determine $u=u^{(N)} \in S_{m-1}^{(-1)}\left(\Pi_{N}\right)$ by the collocation method from the following conditions:

$$
u\left(t_{n j}\right)=\int_{0}^{b} K\left(t_{n j}, s\right) u(s) d s+f\left(t_{n j}\right), \quad j=1, \ldots, m ; \quad n=1, \ldots, N .
$$


If $\eta_{1}=0$, then by $u\left(t_{n 1}\right)$ we denote the right limit $\lim _{t \rightarrow t_{n-1}+0} u(t)$. Similarly, if $\eta_{m}=1$, then $u\left(t_{n m}\right)$ denotes the left limit $\lim _{t \rightarrow t_{n}-0} u(t)$.

\section{A Discrete Collocation Method for Solving Integral Equations}

In order to discretize the integrals in (2.1) we introduce a set of points $S=$ $\left\{s_{j}, j=-M, \ldots, M\right\}$, where $s_{j}=\operatorname{sgn}(j) b(|j| / M)^{\rho}, M>1$ is a natural number, $\operatorname{sgn}(j)=\frac{j}{\max (|j|, 1)}$ and $\rho \geq 1$ is a real number that determines the nonuniformity of the set of points at zero. Next, for a given $t \in[0, b]$ we divide the interval $[0, b]$ into subintervals with the points

$$
V_{t}=\left(\Pi_{N} \cup(t+S)\right) \cap[0, b],
$$

where $t+S=\{t+s: s \in S\}$. Let us number the points in $V_{t}$ in the increasing order so that the index of $t$ is 0 :

$$
V_{t}=\left\{v_{i}: i=-k_{1},-k_{1}+1, \ldots, k_{2}-1, k_{2}\right\},
$$

where

$$
0=v_{-k_{1}}<v_{-k_{1}+1}<\ldots<v_{0}=t<v_{1}<\ldots<v_{k_{2}}=b,
$$

and denote $\Delta v_{i}=v_{i}-v_{i-1}, i=-k_{1}+1, \ldots, k_{2}$. Additionally, we choose a quadrature formula

$$
\int_{0}^{1} x(s) d s \approx \sum_{p=1}^{\ell} w_{p} x\left(\xi_{p}\right)
$$

with knots $0 \leq \xi_{1}<\xi_{2}<\ldots<\xi_{\ell} \leq 1$ and weights $w_{1}, \ldots, w_{\ell}$ and denote

$$
v_{i p}=v_{i-1}+\xi_{p} \Delta v_{i}, i=-k_{1}+1, \ldots k_{2}, p=1, \ldots, \ell .
$$

If $K$ is bounded and has left and right limits as $s \rightarrow t$, then we replace the integrals in (2.1) by the quadrature sums

$$
A_{t} u=\sum_{i=-k_{1}+1}^{k_{2}} \Delta v_{i} \sum_{p=1}^{\ell} w_{p} K\left(t, v_{i p}\right) u\left(v_{i p}\right),
$$

otherwise we use the approximation

$$
A_{t} u=\sum_{i: v_{i-1}<t+s_{-1}} \Delta v_{i} \sum_{p=1}^{\ell} w_{p} K\left(t, v_{i p}\right) u\left(v_{i p}\right)+\sum_{i: v_{i-1} \geq t+s_{1}} \Delta v_{i} \sum_{p=1}^{\ell} w_{p} K\left(t, v_{i p}\right) u\left(v_{i p}\right) .
$$

Our fully discrete collocation method is as follows: we determine an approximate solution $\bar{u} \in S_{m-1}^{(-1)}$ by the condition

$$
\bar{u}\left(t_{n j}\right)=A_{t_{n j}} \bar{u}+f\left(t_{n j}\right), \quad j=1, \ldots, m ; \quad n=1, \ldots, N .
$$




\section{Convergence of the Fully Discrete Method (3.2)}

First we derive an estimate for the error of the quadrature approximation of the integral operator.

Theorem 1. Assume that the quadrature formula (3.1) is exact for all polynomials of order $\mu$, where $\mu \geq m-1$. Assume also, that the kernel $K$ of the integral operator of the equation (1.1) is $\mu-m+2$ times continuously differentiable with respect to $s$ on the set $\{(t, s) \in[0, b] \times[0, b]: t \neq s\}$ and satisfies for $i=0,1, \ldots, \mu-m+2$ the estimates

$$
\left|\frac{\partial^{i} K}{\partial s^{i}}(t, s)\right| \leq c \kappa_{i}(|t-s|),
$$

where

$$
\kappa_{i}(\tau)=\left\{\begin{array}{ll}
1, & i<-\nu, \\
1-\ln \frac{\tau}{b}, & i=-\nu, \\
\tau^{-i-\nu}, & i>-\nu,
\end{array} \quad \tau \in(0, b]\right.
$$

and $\nu<1$ is a real number. Then for all $t \in[0, b]$ we have

$$
\left|\int_{0}^{b} K(t, s) u(s) d s-A_{t} u\right| \leq c\|u\|_{\infty} \begin{cases}M^{-\rho(1-\nu)}, & \rho<\frac{\mu-m+2}{1-\nu}, \nu \notin \mathbb{N} \\ M^{-\rho(1-\nu)}(1+\ln M), & \rho<\frac{\mu-m+2}{1-\nu}, \nu \in \mathbb{N} \\ M^{-\mu+m-2}(1+\ln M), & \rho=\frac{\mu-m+2}{1-\nu}, \\ M^{-\mu+m-2}, & \rho>\frac{\mu-m+2}{1-\nu},\end{cases}
$$

where $c$ does not depend on $t, M, N$ and the form of $\Pi_{N}$ and $\|\cdot\|_{\infty}$ is the $L^{\infty}$ norm.

Proof. Denote

$$
\varepsilon_{i}=\int_{v_{i-1}}^{v_{i}} K(t, s) u(s) d s-\Delta v_{i} \sum_{p=1}^{\ell} w_{p} K\left(t, v_{i p}\right) u\left(v_{i p}\right) .
$$

In the case $\nu \geq 0$, when $K$ may be unbounded, we have

$$
\left|\int_{0}^{b} K(t, s) u(s) d s-A_{t} u\right| \leq \sum_{i: v_{i-1}<t+s_{-1}}\left|\varepsilon_{i}\right|+\|u\|_{\infty} \int_{t+s_{-1}}^{t+s_{1}}|K(t, s)| d s+\sum_{i: v_{i-1} \geq t+s_{1}}\left|\varepsilon_{i}\right| .
$$

If $\nu<0$, then we have

$$
\begin{aligned}
\left|\int_{0}^{b} K(t, s) u(s) d s-A_{t} u\right| & \leq \sum_{i=-k_{1}+1}^{k_{2}}\left|\varepsilon_{i}\right| \leq \sum_{i: v_{i-1} \leq t+s_{-1}}\left|\varepsilon_{i}\right| \\
& +\sum_{i:\left[v_{i-1}, v_{i}\right] \subset\left[t+s_{-1}, t+s_{1}\right]}\left|\varepsilon_{i}\right|+\sum_{i: v_{i-1} \geq t+s_{1}}\left|\varepsilon_{i}\right| .
\end{aligned}
$$


Consider first the common terms (the first and the third) in estimates (4.1) and (4.2). Notice that, since the quadrature formula (3.1) is exact for all polynomials or order $\mu$, we have for all polynomials $\phi$ of order $\mu-m+1$ equality

$$
\varepsilon_{i}=\int_{v_{i-1}}^{v_{i}}(K(t, s)-\phi(s)) u(s) d s-\Delta v_{i} \sum_{p=1}^{\ell} w_{p}\left(K\left(t, v_{i p}\right)-\phi\left(v_{i p}\right)\right) u\left(v_{i p}\right),
$$

thus for any such polynomial $\phi$ we get an estimate

$$
\left|\varepsilon_{i}\right| \leq c\|u\|_{\infty} \Delta v_{i} \sup _{s \in\left[v_{i-1}, v_{i}\right]}|K(t, s)-\phi(s)| .
$$

For values of $i$ such that $v_{i-1}<t+s_{-1}$ we use

$$
\phi(s)=\sum_{q=0}^{\mu-m+1} \frac{1}{q !} \frac{\partial K}{\partial s}\left(t, v_{i}\right)\left(s-v_{i}\right)^{q}
$$

which, together with the well-known estimate for Taylor expansions

$$
|K(t, s)-\phi(s)| \leq \frac{\left|s-v_{i}\right|^{\mu-m+2}}{(\mu-m+2) !} \sup _{s \in\left[v_{i-1}, v_{i}\right]}\left|\frac{\partial^{\mu-m+2} K}{\partial s^{\mu-m+2}}(t, s)\right|
$$

and the assumptions of the theorem gives us

$$
\begin{aligned}
\left|\varepsilon_{i}\right| & \leq c_{1}\|u\|_{\infty}\left(\Delta v_{i}\right)^{\mu-m+3} \sup _{s \in\left[v_{i-1}, v_{i}\right]}\left|\kappa_{\mu-m+2}(|t-s|)\right| \\
& =c_{1}\|u\|_{\infty}\left(\Delta v_{i}\right)^{\mu-m+3} \kappa_{\mu-m+2}\left(\left|t-v_{i}\right|\right)
\end{aligned}
$$

Denote $\Delta s_{j}=s_{j}-s_{j-1}, j=-M+1, \ldots, M$. Then we get an estimate

$$
\begin{aligned}
& \sum_{i: v_{i-1}<t+s_{-1}}\left|\varepsilon_{i}\right|=\sum_{j=-M+1}^{-1} \sum_{i:\left[v_{i-1}, v_{i}\right] \subset\left[t+s_{j-1}, t+s_{j}\right]}\left|\varepsilon_{i}\right| \\
& \leq \sum_{j=-M+1}^{-1} \sum_{i:\left[v_{i-1}, v_{i}\right] \subset\left[t+s_{j-1}, t+s_{j}\right]} c_{1}\|u\|_{\infty}\left(\Delta v_{i}\right)^{\mu-m+3} \kappa_{\mu-m+2}\left(\left|t-v_{i}\right|\right) \\
& \leq c_{1}\|u\|_{\infty} \sum_{j=-M+1}^{-1} \kappa_{\mu-m+2}\left(\left|s_{j}\right|\right) \sum_{i:\left[v_{i-1}, v_{i}\right] \subset\left[t+s_{j-1}, t+s_{j}\right]}\left(\Delta v_{i}\right)^{\mu-m+3} \\
& \left.\leq c_{1}\|u\|_{\infty} \sum_{j=-M+1}^{-1} \sum_{i:\left[v_{i-1}, v_{i}\right] \subset\left[t+s_{j-1}, t+s_{j}\right]} \Delta v_{i}\right)^{\mu-m+3} \\
& \leq c_{1}\|u\|_{\infty} \sum_{j=-M+1}^{-1}\left(\Delta s_{j}\right)^{\mu-m+3} \kappa_{\mu-m+2}\left(\left|s_{j}\right|\right) \\
& \quad=c_{1}\|u\|_{\infty} \sum_{j=1}^{M-1}\left(\Delta s_{j+1}\right)^{\mu-m+3} \kappa_{\mu-m+2}\left(s_{j}\right) .
\end{aligned}
$$


For values of $i$ such that $v_{i-1} \geq t+s_{1}$ we use the estimate (4.3) with $\phi$ that is the Taylor's expansion of $K$ with respect to $s$ at the point $s=v_{i-1}$. Repeating the steps we used for negative values of $i$ we arrive to the same estimate:

$$
\sum_{i: v_{i-1} \geq t+s_{1}}\left|\varepsilon_{i}\right| \leq c_{1}\|u\|_{\infty} \sum_{j=1}^{M-1}\left(\Delta s_{j+1}\right)^{\mu-m+3} \kappa_{\mu-m+2}\left(s_{j}\right) .
$$

Notice that for $j \geq 1$ we have $\Delta s_{j}=b \int_{(j-1) / M}^{j / M} \rho x^{\rho-1} d x$, therefore

$$
\frac{b \rho}{M}\left(\frac{j-1}{M}\right)^{\rho-1} \leq \Delta s_{j} \leq \frac{b \rho}{M}\left(\frac{j}{M}\right)^{\rho-1} \leq \frac{\rho b}{M}
$$

If $\mu-m+2+\nu<1$, then $\kappa_{\mu-m+2}$ is integrable on the interval $[0, b]$ and hence

$$
\begin{gathered}
\sum_{j=1}^{M-1}\left(\Delta s_{j+1}\right)^{\mu-m+3} \kappa_{\mu-m+2}\left(s_{j}\right) \leq\left(\frac{\rho b}{M}\right)^{\mu-m+2} \sum_{j=1}^{M-1} \frac{\Delta s_{j+1}}{\Delta s_{j}} \Delta s_{j} \kappa_{\mu-m+2}\left(s_{j}\right) \\
\leq\left(\frac{\rho b}{M}\right)^{\mu-m+2} \sum_{j=1}^{M-1} \Delta s_{j} \kappa_{\mu-m+2}\left(s_{j}\right)\left\{\begin{array}{l}
\frac{s_{2}}{s_{1}}, \quad \text { if } j=1, \\
\left(\frac{j+1}{j-1}\right)^{\rho-1}, \quad \text { if } j \geq 2
\end{array}\right. \\
\leq\left(\frac{\rho b}{M}\right)^{\mu-m+2} \max \left\{2^{\rho}, 3^{\rho-1}\right\} \sum_{j=1}^{M-1} \Delta s_{j} \kappa_{\mu-m+2}\left(s_{j}\right) \\
\leq\left(\frac{\rho b}{M}\right)^{\mu-m+2} \max \left\{2^{\rho}, 3^{\rho-1}\right\} \int_{0}^{b} \kappa_{\mu-m+2}(s) d s \leq c M^{-\mu+m-2} .
\end{gathered}
$$

If $\mu-m+2+\nu \geq 1$, then

$$
\begin{aligned}
& \sum_{j=1}^{M-1}\left(\Delta s_{j+1}\right)^{\mu-m+3} \kappa_{\mu-m+2}\left(s_{j}\right) \leq \sum_{j=1}^{M-1}\left(\frac{b \rho}{M}\left(\frac{j+1}{M}\right)^{\rho-1}\right)^{\mu-m+3} s_{j}^{-\mu+m-2-\nu} \\
& \leq c M^{-\mu+m-3} \sum_{j=1}^{M-1}\left(\frac{2 j}{M}\right)^{(\rho-1)(\mu-m+3)}\left(\frac{j}{M}\right)^{\rho(-\mu+m-2-\nu)} \\
& \leq c_{1} M^{-\rho(1-\nu)} \sum_{j=1}^{M-1} j^{\rho(1-\nu)-(\mu-m+3)} \\
& \leq c_{1} \begin{cases}M^{-\rho(1-\nu)} \sum_{j=1}^{\infty} j^{\rho(1-\nu)-(\mu-m+3)} & \text { if } \rho(1-\nu)<\mu-m+2, \\
M^{-\rho(1-\nu)}\left(1+\int_{1}^{M} \frac{d \tau}{\tau}\right), & \text { if } \rho(1-\nu)=\mu-m+2, \\
M^{-\rho(1-\nu)} \int_{0}^{M} \tau^{\rho(1-\nu)-(\mu-m+3)} d \tau, & \text { if } \rho(1-\nu)>\mu-m+2\end{cases}
\end{aligned}
$$




$$
\leq c_{2} \begin{cases}M^{-\rho(1-\nu)}, & \text { if } \rho<\frac{\mu-m+2}{1-\nu} \\ M^{-\rho(1-\nu)}(1+\ln M), & \text { if } \rho=\frac{\mu-m+2}{1-\nu} \\ M^{-\mu+m-2}, & \text { if } \rho>\frac{\mu-m+2}{1-\nu}\end{cases}
$$

Thus we have shown for all values of $\nu, \mu$ and $m$ that

$$
\sum_{i: v_{i-1}<t+s_{-1}}\left|\varepsilon_{i}\right|+\sum_{i: v_{i-1} \geq t+s_{1}}\left|\varepsilon_{i}\right| \leq c \begin{cases}M^{-\rho(1-\nu)}, & \text { if } \rho<\frac{\mu-m+2}{1-\nu} \\ M^{-\rho(1-\nu)}(1+\ln M), & \text { if } \rho=\frac{\mu-m+2}{1-\nu} \\ M^{-\mu+m-2}, & \text { if } \rho>\frac{\mu-m+2}{1-\nu}\end{cases}
$$

It remains to estimate the middle terms of (4.1) and (4.2). If $\nu>0$, then the middle term in (4.1) can be estimated easily:

$$
\begin{aligned}
\|u\|_{\infty} \int_{t+s_{-1}}^{t+s_{1}}|K(t, s)| d s & \leq c\|u\|_{\infty} \int_{t+s_{-1}}^{t+s_{1}}|t-s|^{-\nu} d s=\|u\|_{\infty} \frac{2 c}{1-\nu}\left(\frac{b}{M^{\rho}}\right)^{1-\nu} \\
& \leq c_{1}\|u\|_{\infty} M^{-\rho(1-\nu)}
\end{aligned}
$$

If $\nu=0$ then

$$
\begin{aligned}
\|u\|_{\infty} \int_{t+s_{-1}}^{t+s_{1}}|K(t, s)| d s & \leq c\|u\|_{\infty} \int_{s_{-1}}^{s_{1}}\left(1-\ln \frac{|\tau|}{b}\right) d \tau \\
& \leq c_{1}\|u\|_{\infty} M^{-\rho}(1+\ln M) .
\end{aligned}
$$

In both cases the estimate of the middle term on the right hand side of (4.1) agrees well with (i.e. it is bounded from above by) the estimate of the Theorem, so for $\nu \geq 0$ the Theorem is proved.

If $\nu<0$, then we divide the middle term of (4.2) into two halves:

$$
\sum_{i:\left[v_{i-1}, v_{i}\right] \subset\left[t+s_{-1}, t+s_{1}\right]}\left|\varepsilon_{i}\right|=\sum_{i:\left[v_{i-1}, v_{i}\right] \subset\left[t+s_{-1}, t\right]}\left|\varepsilon_{i}\right|+\sum_{i:\left[v_{i-1}, v_{i}\right] \subset\left[t, t+s_{1}\right]}\left|\varepsilon_{i}\right| .
$$

Since the estimation procedure is the same for both halves, we deal carefully with the the second one only. Let $q$ be the largest integer such that $q \leq \mu-m+1$ and $q+\nu<0$, then the derivatives $\frac{\partial^{j} K}{\partial s^{j}}(t, s)$ have finite right (and left) limits as $s \rightarrow t$ for $j=0,1, \ldots, q$. We denote

$$
\frac{\partial^{j} K}{\partial s^{j}}(t, t+)=\lim _{s \rightarrow t, s>t} \frac{\partial^{j} K}{\partial s^{j}}(t, s), \quad j=0,1, \ldots, q
$$

and define

$$
\phi(s)=\sum_{j=0}^{q} \frac{1}{j !} \frac{\partial^{j} K}{\partial s^{j}}(t, t+)(s-t)^{j} .
$$

Using now the integral representation of the error of the Taylor's expansion

$$
K(t, s)-\phi(s)=\frac{1}{q !} \int_{t}^{s}(s-\tau)^{q} \frac{\partial^{q+1} K}{\partial s^{q+1}}(t, \tau) d \tau
$$


and the estimate (4.3) we get

$$
\left|\varepsilon_{i}\right| \leq c\|u\|_{\infty} \Delta v_{i} \frac{s_{1}^{q}}{q !} \int_{t}^{t+s_{1}} \kappa_{q+1}(\tau-t) d \tau
$$

SO

$$
\sum_{i:\left[v_{i-1}, v_{i}\right] \subset\left[t, t+s_{1}\right]}\left|\varepsilon_{i}\right| \leq c \frac{\left(b / M^{\rho}\right)^{q+1}}{q !} \int_{0}^{s_{1}} \kappa_{q+1}(\tau) d \tau .
$$

If $q+1>-\nu$, then

$$
\int_{0}^{s_{1}} \kappa_{q+1}(\tau) d \tau=\int_{0}^{s_{1}} \tau^{-q-1-\nu} d \tau \leq c M^{\rho(q+\nu)},
$$

in the case $q+1=-\nu$ we have

$$
\int_{0}^{s_{1}} \kappa_{q+1}(\tau) d \tau=\int_{0}^{s_{1}}\left(1-\ln \frac{\tau}{b}\right) d \tau \leq c M^{-\rho}(1+\ln M),
$$

thus

$$
\sum_{i:\left[v_{i-1}, v_{i}\right] \subset\left[t, t+s_{1}\right]}\left|\varepsilon_{i}\right| \leq c \begin{cases}M^{-\rho(1-\nu)}, & \text { if } \nu \notin \mathbb{N}, \\ M^{-\rho(1-\nu)}(1+\ln M), & \text { if } \nu \in \mathbb{N} .\end{cases}
$$

Since the last estimate is clearly bounded above by the estimate of the Theorem, the proof is completed for all values of $\nu$.

The previous technical result enables us to estimate easily the difference of solutions of the exact collocation method (2.1) and the fully discrete collocation method (3.2).

Theorem 2. Assume that the integral equation (1.1) is uniquely solvable in $L^{\infty}$ and that a sequence of grids $\Pi_{N}$ such that the length of the maximal subinterval of $\Pi_{N}$ goes to 0 as $N \rightarrow \infty$ is given. Assume also that the collocation method (2.1) is used and that the quadrature formula (3.1) is exact for all polynomials of order $\mu$, where $\mu \geq m-1$. Assume also, that the kernel $K$ of the integral operator of the equation (1.1) is continuous and $\mu-m+2$ times continuously differentiable with respect to $s$ on the set $\{(t, s) \in[0, b] \times[0, b]: t \neq s\}$ and satisfies the estimates of Theorem 1. Then there exist integers $N_{0}$ and $M_{0}$ such that for all $N \geq N_{0}$ and for all $M \geq M_{0}$ both methods (2.1) and (3.2) are uniquely solvable and the solutions $u$ of (2.1) and $\bar{u}$ of (3.2) satisfy the inequality

$$
\|u-\bar{u}\|_{\infty} \leq c\left\{\begin{array}{ll}
M^{-\rho(1-\nu)}, & \rho<\frac{\mu-m+2}{1-\nu}, \nu \notin \mathbb{N} \\
M^{-\rho(1-\nu)}(1+\ln M), & \rho<\frac{\mu-m+2}{1-\nu}, \nu \in \mathbb{N} \\
M^{-\mu+m-2}(1+\ln M), & \rho=\frac{\mu-m+2}{1-\nu}, \\
M^{-\mu+m-2}, & \rho>\frac{\mu-m+2}{1-\nu},
\end{array},\right.
$$

where the constant $c$ does not depend on $M$ and $N$. 
Proof. Define an operator $T: L^{\infty}(0, b) \rightarrow C[0, b]$ by $T x(t)=\int_{0}^{b} K(t, s) x(s) d s$ and a piecewise polynomial interpolation operator $P_{N}: C[0, b] \rightarrow S_{m-1}^{(-1)}\left(\Pi_{N}\right) \subset$ $L^{\infty}(0, b)$ by conditions

$$
P_{N} x\left(t_{n j}\right)=x\left(t_{n j}\right), n=1, \ldots, N, j=1, \ldots, m .
$$

It is well known that $T$ is a compact operator and that $P_{N}$ is a sequence of uniformly bounded operators satisfying

$$
\left\|P_{N} x-x\right\|_{\infty} \rightarrow 0 \text { for all } x \in C[0, b] .
$$

Therefore $\left\|P_{N} T-T\right\|_{\mathcal{L}\left(L^{\infty}(0, b)\right)} \rightarrow 0$ as $N \rightarrow \infty$ (for a detailed proof of this result see [5], Lemma 2.4.6) and hence (since $I-T$ is invertible) there exists $N_{0}$ such that for every $N \geq N_{0}$ the estimate

$$
\left\|\left(I-P_{N} T\right)^{-1}\right\|_{\mathcal{L}\left(L^{\infty}(0, b)\right)} \leq 2\left\|(I-T)^{-1}\right\|_{\mathcal{L}\left(L^{\infty}(0, b)\right)}
$$

holds. Denote by $X_{N}$ the space of piecewise polynomial functions $S_{m-1}^{(-1)}\left(\Pi_{N}\right)$ equipped with $L^{\infty}(0, b)$ norm. Define an operator $\tilde{T}_{N, M}: X_{N} \rightarrow X_{N}$ by the interpolation conditions

$$
\tilde{T}_{N, M} x\left(t_{n j}\right)=A_{t_{n j}} x, n=1, \ldots, N, j=1, \ldots, m, x \in X_{N},
$$

then

$$
\left\|\left(P_{N} T-\tilde{T}_{N, M}\right) x\right\|_{\infty} \leq c \max _{n=1, \ldots, N, j=1, \ldots, m}\left\{\left|A_{t_{n j}} x-T x\left(t_{n j}\right)\right|\right\}
$$

where the constant $c$ depends only on $m$ and the choice of collocation parameters $\eta_{1}, \ldots, \eta_{m}$. Thus, according to Theorem 1 we have

$$
\left\|P_{N} T-\tilde{T}_{N, M}\right\|_{\mathcal{L}\left(X_{N}\right)} \leq c \begin{cases}M^{-\rho(1-\nu)}, & \rho<\frac{\mu-m+2}{1-\nu}, \nu \notin \mathbb{N}, \\ M^{-\rho(1-\nu)}(1+\ln M), & \rho<\frac{\mu-m+2}{1-\nu}, \nu \in \mathbb{N}, \\ M^{-\mu+m-2}(1+\ln M), & \rho=\frac{\mu-m+2}{1-\nu}, \\ M^{-\mu+m-2}, & \rho>\frac{\mu-m+2}{1-\nu} .\end{cases}
$$

Let us choose $M_{0}$ such that for $M \geq M_{0}$ we have

$$
\left\|P_{N} T-\tilde{T}_{N, M}\right\|_{\mathcal{L}\left(X_{N}\right)} \leq \frac{1}{4\|I-T\|_{\mathcal{L}^{\infty}(0, b)}} .
$$

Notice that the numerical methods (2.1) and (3.2) correspond to operator equations

$$
u=P_{N} T u+P_{N} f, \quad \bar{u}=\tilde{T}_{N, M} \bar{u}+P_{N} f
$$

in the space $X_{N}$, respectively. Denote $B=I-P_{N} T, \delta B=P_{N} T-\tilde{T}_{N, M}$ and $\delta u=u-\bar{u}$, then the equations take forms

$$
B u=P_{N} f,(B+\delta B)(u+\delta u)=P_{N} f .
$$


According to our choices of $N_{0}$ and $M_{0}$ the operators $B$ and $B+\delta B$ are both invertible for all $N \geq N_{0}, M \geq M_{0}$ and satisfy estimates

$$
\begin{aligned}
& \left\|B^{-1}\right\|_{\mathcal{L}\left(X_{N}\right)} \leq 2\left\|(I-T)^{-1}\right\|_{\mathcal{L}\left(L^{\infty}(0, b)\right)}, \\
& \left\|(B+\delta B)^{-1}\right\|_{\mathcal{L}\left(X_{N}\right)} \leq \frac{\left\|B^{-1}\right\|_{\mathcal{L}\left(X_{N}\right)}}{1-\left\|B^{-1}\right\|_{\mathcal{L}\left(X_{N}\right)}\|\delta B\|_{\mathcal{L}\left(X_{N}\right)}} \leq 4\left\|(I-T)^{-1}\right\|_{\mathcal{L}\left(L^{\infty}(0, b)\right)}
\end{aligned}
$$

therefore

$$
\begin{aligned}
\|u-\bar{u}\|_{\infty} & =\|\delta u\|_{\infty}=\left\|\left[(B+\delta B)^{-1}-B^{-1}\right] P_{N} f\right\|_{\infty} \\
& =\left\|B^{-1} \delta B(B+\delta B)^{-1} P_{N} f\right\|_{\infty} \leq c\|\delta B\|_{\mathcal{L}\left(X_{N}\right)}=\left\|P_{N} T-\tilde{T}_{N, M}\right\|_{\mathcal{L}\left(X_{N}\right)}
\end{aligned}
$$

where $c$ does not depend on $M$ and $N$. Taking into account the estimate (4.4), the statement of Theorem 2 is proved.

Theorem 2 tells us that for any collocation method we can choose appropriate $M$ depending on $N$ (for example $M=N$ ), an appropriate quadrature formula (3.1) and a suitable value for the nonuniformity parameter $\rho$ so that the convergence rate of the fully discrete collocation method is of the same order as the convergence rate of the collocation method.

\section{References}

[1] H. Brunner. The numerical solution of weakly singular Volterra integral equations by collocation on graded meshes. Math. Comp., 45:417-437, 1985.

[2] H. Brunner, A. Pedas and G. Vainikko. The piecewise polynomial collocation method for nonlinear weakly singular Volterra equations. Math. Comp., 68(227):1079-1095, 1999.

[3] H. Kaneko and Y. Xu. Gauss-Type quadratures for weakly singular integrals and their application to Fredholm integral equations of second kind. Math. Comp., 62:739-753, 1994.

[4] R. Kangro and I. Kangro. On the stability of piecewise polynomial collocation methods for solving weakly singular integral equations of the second kind. Math. Model. Anal., 13(1):29-36, 2008. (doi:10.3846/1392-6292.2008.13.29-36)

[5] I. Parts. Piecewise polynomial collocation methods for solving weakly singular integro-differential equations. PhD thesis, 2005. Available from Internet: http://www.utlib.ee/ekollekt/diss/dok/2005/b1741779x/parts.pdf [cited 30 September 2008].

[6] A. Pedas and G. Vainikko. Superconvergence of piecewise polynomial collocation for nonlinear weakly singular integral equations. J. Integral Equations Appl., 9:379-406, 1997.

[7] E. Tamme. Fully discrete collocation method for weakly singular integral equations. Proc. Estonian Acad. Sci. Phys. Math., 50(3):133-144, 2001.

[8] G. Vainikko. Multidimensional Weakly Singular Integral Equations, Lecture Notes Math. Springer-Verlag, Berlin-Heidelberg-New York, 1993.

[9] G. Vainikko, A. Pedas and P. Uba. Methods for Solving Weakly Singular Integral Equations. Univ. of Tartu, Tartu, 1984. (in Russian) 